\title{
Embedding of post-Lie algebras into postassociative algebras V. Gubarev
}

In honour of 80th anniversary of Professor Leonid Arkad'evich Bokut'

\begin{abstract}
Applying Gröbner-Shirshov technique, we prove that any post-Lie algebra injectively embeds into its universal enveloping postassociative algebra.

Keywords: Rota-Baxter algebra, postassociative algebra, post-Lie algebra, Gröbner-Shirshov bases.
\end{abstract}

\section{Introduction}

Linear operator $R$ defined on an algebra $A$ over the field $\mathbb{k}$ is called a Rota-Baxter operator (RB-operator, for short) of a weight $\lambda \in \mathbb{k}$ if it satisfies the relation

$$
R(x) R(y)=R(R(x) y+x R(y)+\lambda x y), \quad x, y \in A .
$$

In this case, an algebra $A$ is called Rota-Baxter algebra (RB-algebra).

G. Baxter defined the notion of what is now called Rota-Baxter operator on a (commutative) algebra in 1960 [4], solving an analytic problem. The relation (11) with $\lambda=0$ appeared as a generalization of integration by parts formula. G.-C. Rota [34], P. Cartier [7] and others studied different combinatorial properties of RB-operators and RB-algebras. In 1980s, the deep connection between Lie RB-algebras and Yang-Baxter equation was found [5, 35].

There are different constructions of the free commutative RB-algebra [34, 7, 24]. In 2008, K. Ebrahimi-Fard and L. Guo constructed the free associative RB-algebra [12]. In 2010, L.A. Bokut, Yu. Chen and X. Deng [6] found a linear basis of the free associative RB-algebra by means of Gröbner-Shirshov technique. Linear bases of the free Lie RBalgebra were found in [16, 22, 33].

Pre-Lie algebras were introduced independently by E.B. Vinberg [37] in 1960 and M. Gerstenhaber [13] in 1963. Pre-Lie algebras also known as left-symmetric algebras satisfy the identity $\left(x_{1} x_{2}\right) x_{3}-x_{1}\left(x_{2} x_{3}\right)=\left(x_{2} x_{1}\right) x_{3}-x_{2}\left(x_{1} x_{3}\right)$.

In 2001, J.-L. Loday [28] defined the dendriform dialgebra (preassociative algebra) as a vector space endowed with two bilinear operations $\succ, \prec$ satisfying

$$
\begin{aligned}
\left(x_{1} \succ x_{2}+x_{1} \prec x_{2}\right) & \succ x_{3}=x_{1} \succ\left(x_{2} \succ x_{3}\right),\left(x_{1} \succ x_{2}\right) \prec x_{3}=x_{1} \succ\left(x_{2} \prec x_{3}\right), \\
x_{1} & \prec\left(x_{2} \succ x_{3}+x_{2} \prec x_{3}\right)=\left(x_{1} \prec x_{2}\right) \prec x_{3} .
\end{aligned}
$$

In 1995, [27] J.-L. Loday also defined Zinbiel algebra (precommutative algebra), on which the identity $\left(x_{1} \succ x_{2}+x_{2} \succ x_{1}\right) \succ x_{3}=x_{1} \succ\left(x_{2} \succ x_{3}\right)$ holds. Any preassociative algebra with the identity $x \succ y=y \prec x$ is a precommutative algebra and under the product $x \cdot y=x \succ y-y \prec x$ is a pre-Lie algebra.

In 2004, dendriform trialgebra (postassociative algebra) was introduced [31], i.e., an algebra with bilinear operations $\prec, \succ$, satisfying seven certain axioms. A space $A$ with 
two bilinear operations [,] and · is called a post-Lie algebra (Vallette, 2007 [36]) if [,] is a Lie bracket and the next identities hold

$$
(x \cdot y) \cdot z-x \cdot(y \cdot z)-(y \cdot x) \cdot z+y \cdot(x \cdot z)=[y, x] \cdot z, \quad x \cdot[y, z]=[x \cdot y, z]+[y, x \cdot z] .
$$

Let us explain the choice of terminology. Given a binary quadratic operad $\mathcal{P}$, the defining identities for pre- and post- $\mathcal{P}$-algebras were found in [2]. One can define the operad of pre- and post- $\mathcal{P}$-algebras as $\mathcal{P} \bullet$ PreLie and $\mathcal{P} \bullet$ PostLie respectively. Here PreLie and PostLie denote the operads of pre-Lie algebras and post-Lie algebras respectively, denotes the black Manin product of operads [14]. By pre- or postalgebra we will mean pre- or post- $\mathcal{P}$-algebra for some operad $\mathcal{P}$.

In 2000, M. Aguiar [1] stated that any associative (commutative) algebra with a given Rota-Baxter operator $R$ of weight zero under the operations $a \succ b=R(a) b$, $a \prec b=a R(b)$ is a preassociative (precommutative) algebra. In 2002, K. EbrahimiFard [11] showed that any associative RB-algebra of nonzero weight $\lambda$ under the same two products $\succ, \prec$ and the third operation $a \cdot b=\lambda a b$ is a postassociative algebra. Analogous statement for postcommutative algebras was obtained by J.-L. Loday in 2007 [29]. The analogue of the Aguiar construction for the pair of pre-Lie algebras and Lie RB-algebras of weight zero was stated in 2000 by M. Aguiar [1] and by I.Z. Golubchik, V.V. Sokolov [15]. In 2010 [3], this construction for the pair of post-Lie algebras and Lie RB-algebras of nonzero weight was extended.

In 2013 [2], the construction of M. Aguiar and K. Ebrahimi-Fard was generalized for the case of arbitrary variety.

In 2008, the notion of universal enveloping RB-algebras of pre- and postassociative algebras was introduced [12. In [12], it was also proved that the universal enveloping of a free preassociative algebra is free.

In 2010, with the help of Gröbner-Shirshov bases [6], Yu. Chen and Q. Mo proved that any preassociative algebra over the field of characteristic zero injectively embeds into its universal enveloping RB-algebra [8].

In 2013 [20], given a variety Var, it was proved that every pre-Var-algebra (post-Varalgebra) injectively embeds into its universal enveloping Var-RB-algebra of weight $\lambda=0$ $(\lambda \neq 0)$. Based on the last result, we formulate

Problem 1. Construct the universal enveloping RB-algebra of a pre- or postalgebra.

In [23], L. Guo actually stated the following problem for Var = As:

Problem 2. Clarify if the pairs of varieties (RBVar, preVar) and ( $\mathrm{RB}_{\lambda}$ Var, postVar) for $\lambda \neq 0$ are Poincaré - Birkhoff-Witt (PBW-) pairs in the sense of [32].

Here RBVar $\left(\mathrm{RB}_{\lambda}\right.$ Var) denotes the variety of Var-algebras endowed with an RBoperator of (non)zero weight $\lambda$.

Problem 3. (Guo et al., [25]) Given a variety Var of algebras, whether the variety of RB-Var-algebras is Schreier, i.e., whether every subalgebra of the free algebra is free itself?

Problem 4. a) Prove that any pre-Lie (post-Lie) algebra injectively embeds into its universal enveloping preassociative (postassociative) algebra.

b) Construct the universal enveloping preassociative (postassociative) algebra for given pre-Lie (post-Lie) algebra. 
The answer on Problem 4b will cover Problem 4a. For pre-Lie algebras, Problem 4b and Problem 2 were stated in [26]. The discussion of Problem 4 in the case of restricted pre-Lie algebras can be found in [9]. The analogues of Problem 4 for Koszul-dual objects, di- and trialgebras, were solved in [30, 21].

Problems 1-3 were solved by author in commutative [17], associative [18], and Lie [19] cases. So, the question of L. Guo [23] is completely answered.

The main result of the current work is the affirmative answer on Problem $4 \mathrm{a}$ in postalgebra case. In April 2018, the proof that the pair of varieties of pre-Lie and preassociative algebras is a PBW-pair was announced [10].

\section{Postalgebras}

A postassociative algebra is a linear space with three bilinear operations $\cdot, \succ, \prec$ satisfying seven identities:

$$
\begin{aligned}
&(x \prec y) \prec z= x \prec(y \succ z+y \prec z+y \cdot z), \quad(x \succ y) \prec z=x \succ(y \prec z), \\
&(x \succ y+y \succ x+x \cdot y) \succ z=x \succ(y \succ z), \\
& x \succ(y \cdot z)=(x \succ y) \cdot z, \quad(x \prec y) \cdot z=x \cdot(y \succ z), \\
&(x \cdot y) \prec z=x \cdot(y \prec z), \quad(x \cdot y) \cdot z=x \cdot(y \cdot z) .
\end{aligned}
$$

A post-Lie algebra is a vector space endowed with two bilinear products [,] and ·, the bracket [,] is Lie, and the following identities are fulfilled:

$(x \cdot y) \cdot z-x \cdot(y \cdot z)-(y \cdot x) \cdot z+y \cdot(x \cdot z)=[y, x] \cdot z, \quad x \cdot[y, z]=[x \cdot y, z]+[y, x \cdot z]$.

\section{Embedding of pre- and postalgebras into RB-algebras}

Theorem 1 [1, 2, 3, 11, 15, 29]. Let $A$ be an RB-algebra of a variety Var and weight $\lambda=0(\lambda \neq 0)$. With respect to the operations

$$
x \succ y=R(x) y, \quad x \prec y=x R(y) \quad(x \cdot y=\lambda x y)
$$

$A$ is a pre-Var-algebra (post-Var-algebra).

Denote the pre- and post-Var-algebra obtained in Theorem 1 as $A_{\lambda}^{(R)}$.

Given a pre-Var-algebra $\langle C, \succ, \prec\rangle$, universal enveloping RB-Var-algebra $U$ of $C$ is the universal algebra in the class of all RB-Var-algebras of weight zero such that there exists homomorphism from $C$ to $U_{0}^{(R)}$. Analogously universal enveloping RB-Var-algebra of a post-Var-algebra is defined.

Theorem 2 [20]. Any pre-Var-algebra (post-Var-algebra) could be embedded into its universal enveloping RB-algebra of the variety Var and weight $\lambda=0(\lambda \neq 0)$.

Let us consider the idea of the proof of Theorem 2 in the postalgebra case. Suppose $\langle A, \succ, \prec, \cdot\rangle$ is a post-Var-algebra. Then the direct sum of two isomorphic copies of $A$, the space $\hat{A}=A \oplus A^{\prime}$, endowed with a binary operation

$$
a * b=a \succ b+a \prec b+a \cdot b, a * b^{\prime}=(a \succ b)^{\prime}, a^{\prime} * b=(a \prec b)^{\prime}, a^{\prime} * b^{\prime}=(\lambda a \cdot b)^{\prime}
$$


for $a, b \in A$, is proved to be an algebra of the variety Var. Moreover, the map $R\left(a^{\prime}\right)=\lambda a$, $R(a)=-\lambda a$ is an RB-operator of weight $\lambda$ on $\hat{A}$. The injective embedding of $A$ into $\hat{A}$ is given by $a \mapsto a^{\prime}, a \in A$. However, $\hat{A}$ is not a universal enveloping RB-algebra of $A$.

\section{Embedding of post-Lie algebras into postassocia- tive algebras}

Let $R \operatorname{As}\langle X\rangle$ denote the free associative algebra generated by a set $X$ with a linear map $R$ in the signature. One can construct a linear basis of $R A s\langle X\rangle$ (see, e.g., [25]) by induction. First, all elements from $S(X)$ lie in the basis. Next, if we have basic elements $a_{1}, a_{2}, \ldots, a_{k}, k \geq 1$, then the word $w_{1} R\left(a_{1}\right) w_{2} \ldots w_{k} R\left(a_{k}\right) w_{k+1}$ lies in the basis of $R A s\langle X\rangle$. Here $w_{1}, \ldots, w_{k+1} \in S(X) \cup \emptyset$. Let us denote the basis obtained as $R S(X)$. Given a word $u$ from $R S(X)$, the number of appeareances of the symbol $R$ in $u$ is denoted by $\operatorname{deg}_{R}(u), R$-degree of $u$. We call any element from $R S(X)$ of the form $R(w)$ as $R$ letter. By $X_{\infty}$ we denote the union of $X$ and the set of all $R$-letters. Given $u \in R S(X)$, define $\operatorname{deg} u$ (degree of $u$ ) as the length of $u$ in the alphabet $X_{\infty}$.

Suppose that $X$ is a well-ordered set with respect to $<$. Let us introduce by induction the deg-lex order on $S(X)$. Firstly, we compare two words $u$ and $v$ by the length: $u<v$ if $|u|<|v|$. Secondly, when $|u|=|v|, u=x_{i} u^{\prime}, v=x_{j} v^{\prime}, x_{i}, x_{j} \in X$, we have $u<v$ if either $x_{i}<x_{j}$ or $x_{i}=x_{j}, u^{\prime}<v^{\prime}$. We compare two words $u$ and $v$ from $R S(X)$ by $R$-degree: $u<v$ if $\operatorname{deg}_{R}(u)<\operatorname{deg}_{R}(v)$. If $\operatorname{deg}_{R}(u)=\operatorname{deg}_{R}(v)$, we compare $u$ and $v$ in deg-lex order as words in the alphabet $X_{\infty}$. Here we define each $x$ from $X$ to be less than any $R$-letter and $R(a)<R(b)$ if and only if $a<b$. Finally, we define an order $<$ on $R \operatorname{As}\langle X\rangle$, extending it from basic elements.

Let $*$ be a symbol not containing in $X$. By a $*$-bracketed word on $X$, we mean any basic word from $R$ As $\langle X \cup\{*\}\rangle$ with exactly one occurrence of $*$. The set of all $*$-bracketed words on $X$ is denoted by $R S^{*}(X)$. For $q \in R S^{*}(X)$ and $u \in R A s\langle X\rangle$, we define $\left.q\right|_{u}$ as $\left.q\right|_{* \rightarrow u}$ to be the bracketed word obtained by replacing the letter $*$ in $q$ by $u$.

The order defined above is monomial, i.e., from $u<v$ follows that $\left.q\right|_{u}<\left.q\right|_{v}$ for all $u, v \in R S(X)$ and $q \in R S^{*}(X)$.

Given $f \in R A s\langle X\rangle$, by $\bar{f}$ we mean the leading word in $f$.

Definition 1 [25]. Let $f, g \in R \operatorname{As}\langle X\rangle$. If there exist $\mu, \nu, w \in R S(X)$ such that $w=\bar{f} \mu=\nu \bar{g}$ with $\operatorname{deg} w<\operatorname{deg}(\bar{f})+\operatorname{deg}(\bar{g})$, then we define $(f, g)_{w}$ as $f \mu-\nu g$ and call it the composition of intersection of $f, g$ with respect to $(\mu, \nu)$. If there exist $q \in R S^{*}(X)$ and $w \in R S(X)$ such that $w=\bar{f}=\left.q\right|_{\bar{g}}$, then we define $(f, g)_{w}^{q}$ as $f-\left.q\right|_{g}$ and call it the composition of inclusion of $f, g$ with respect to $q$.

Definition 2 [25]. Let $S$ be a subset of monic elements from $R \operatorname{As}\langle X\rangle$ and $w \in R S(X)$.

(1) For $u, v \in R \operatorname{As}\langle X\rangle$, we call $u$ and $v$ congruent modulo $(S, w)$ and denote this by $u \equiv v \bmod (S, w)$ if $u-v=\left.\sum c_{i} q_{i}\right|_{s_{i}}$ with $c_{i} \in \mathbb{k}, q_{i} \in R S^{*}(X), s_{i} \in S$ and $\left.q_{i}\right|_{\overline{s_{i}}}<w$.

(2) For $f, g \in R \operatorname{As}\langle X\rangle$ and suitable $w, \mu, \nu$ or $q$ that give a composition of intersection $(f, g)_{w}$ or a composition of inclusion $(f, g)_{w}^{q}$, the composition is called trivial modulo $(S, w)$ if $(f, g)_{w}$ or $(f, g)_{w}^{q} \equiv 0 \bmod (S, w)$.

(3) The set $S \subset R \operatorname{As}\langle X\rangle$ is called a Gröbner-Shirshov basis if, for all $f, g \in S$, all 
compositions of intersection $(f, g)_{w}$ and all compositions of inclusion $(f, g)_{w}^{q}$ are trivial modulo $(S, w)$.

Theorem 3 [25]. Let $S$ be a set of monic elements in $R A s\langle X\rangle$, let $<$ be a monomial ordering on $R S(X)$ and let $I d(S)$ be the $R$-ideal of $R A s\langle X\rangle$ generated by $S$. If $S$ is a Gröbner-Shirshov basis in $R \operatorname{As}\langle X\rangle$, then $R \operatorname{As}\langle X\rangle=\mathbb{k} \operatorname{Irr}(S) \oplus \operatorname{Id}(S)$ where $\operatorname{Irr}(S)=$ $R S(X) \backslash\left\{\left.q\right|_{\bar{s}} \mid q \in R S^{*}(X), s \in S\right\}$ and $\operatorname{Irr}(S)$ is a linear basis of $R \operatorname{As}\langle X\rangle / \operatorname{Id}(S)$.

Let $A$ be an associative algebra with an RB-operator $R$. Then the algebra $A^{(-)}$is a Lie RB-algebra under the product $[x, y]=x y-y x$ and the same action of $R$. So, we state the analogue of Problem 1.4. How does the left adjoint functor from the category of Lie RB-algebras to the category of associative RB-algebras (of the same weight) look like? Is it the embedding always injective?

Let $\hat{L}=L \oplus L^{\prime}$ be exactly the Lie algebra with the RB-operator $R$ of weight -1 constructed in the sketch of the proof of Theorem 2. Then $R\left(y_{\alpha}\right)=y_{\alpha}, R\left(x_{\alpha}\right)=0$, and $X_{\Lambda} \cup Y_{\Lambda}=\left\{x_{\alpha}, \alpha \in \Lambda\right\} \cup\left\{y_{\alpha}, \alpha \in \Lambda\right\}$ is a linear basis of $\hat{L}$. Note that $\operatorname{Span}\{X\}$ and $\operatorname{Span}\{Y\}$ are Lie subalgebras of $\hat{L}$. Suppose that $\Lambda$ is well-ordered set. Extend the order to $X_{\Lambda} \cup Y_{\Lambda}$ as follows: $t_{\alpha}<t_{\beta}, t \in\{x, y\}$, if and only if $\alpha<\beta$ and $y_{\alpha}<x_{\beta}$ for all $\alpha, \beta \in \Lambda$.

Consider the set $S$ of the following elements in $R \operatorname{As}\left\langle X_{\Lambda} \cup Y_{\Lambda}\right\rangle$ :

$$
\begin{gathered}
x_{\alpha} x_{\beta}-x_{\beta} x_{\alpha}-\left[x_{\alpha}, x_{\beta}\right], y_{\alpha} y_{\beta}-y_{\beta} y_{\alpha}-\left[y_{\alpha}, y_{\beta}\right], \beta<\alpha, \\
x_{\alpha} y_{\beta}-y_{\beta} x_{\alpha}-\left[x_{\alpha}, y_{\beta}\right], \\
R\left(\vec{y}_{\alpha}\right)-\vec{y}_{\alpha}, \\
R\left(\vec{x}_{\alpha}\right), \\
R\left(R\left(a_{1}\right) \vec{y}_{\alpha_{1}} R\left(a_{2}\right) \ldots R\left(a_{s}\right) \vec{y}_{\alpha_{s}} R\left(a_{s+1}\right)\right)-R\left(a_{1}\right) \vec{y}_{\alpha_{1}} R\left(a_{2}\right) \ldots R\left(a_{s}\right) \vec{y}_{\alpha_{s}} R\left(a_{s+1}\right), \\
R\left(R(a) \vec{x}_{\alpha} R(b)\right)-R\left(R(a) \vec{x}_{\alpha} b+a \vec{x}_{\alpha} R(b)-a \vec{x}_{\alpha} b\right), \\
R\left(R(a) \vec{x}_{\alpha}\right)-R\left(a \vec{x}_{\alpha}\right), \quad R\left(\vec{x}_{\alpha} R(b)\right)-R\left(\vec{x}_{\alpha} b\right), \\
R(a) R(b)-R(R(a) b+a R(b)-a b) .
\end{gathered}
$$

Here $a, b, a_{i}$ are elements from $R S^{\prime}\left(X_{\Lambda} \cup Y_{\Lambda}\right)=R S\left(X_{\Lambda} \cup Y_{\Lambda}\right) \backslash\left(S\left(X_{\Lambda}\right) \cup S\left(Y_{\Lambda}\right)\right)$. By $\vec{y}_{\alpha_{i}}$ or $\vec{x}_{\alpha}$ we mean a monomial from $\mathbb{k}\left[Y_{\Lambda}\right]$ or $\mathbb{k}\left[X_{\Lambda}\right]$ respectively.

Theorem 4. The set $S$ is a a Gröbner-Shirshov basis in $R \operatorname{As}\left\langle X_{\Lambda} \cup Y_{\Lambda}\right\rangle$.

Proof. It is known that all compositions between two elements from (5) are trivial, as it is the method to construct the classical universal enveloping algebra.

Compositions of intersection between two expressions from (11) are trivial by [25]. Thus, all compositions of intersection which are not at the same time compositions of inclusion are trivial.

Let us consider a composition of inclusion between (17) and (11). Let $\vec{x}_{\alpha} \in C(X)$, $a \in R S^{\prime}\left(X_{\Lambda} \cup Y_{\Lambda}\right), w=R(a) R\left(\vec{x}_{\alpha}\right)$. By the following, we get that the composition of inclusion is trivial:

$$
\begin{aligned}
& R(a) R\left(\vec{x}_{\alpha}\right) \stackrel{(\underline{7})}{=} 0 \bmod (S, w), \\
& R(a) R\left(\vec{x}_{\alpha}\right) \stackrel{(11)}{\equiv} R\left(R(a) \vec{x}_{\alpha}+a R\left(\vec{x}_{\alpha}\right)-a \vec{x}_{\alpha}\right) \stackrel{(10)}{\equiv} R\left(a R\left(\vec{x}_{\alpha}\right)\right) \stackrel{\left(\frac{70}{\equiv}\right.}{=} 0 \bmod (S, w) .
\end{aligned}
$$


A composition of inclusion between (6) and (11) is analogously trivial.

Compute a composition of inclusion between (8) and (11). For $\vec{y}_{\alpha_{i}} \in \mathbb{k}[Y], w=$ $R(b) R\left(R\left(a_{1}\right) \vec{y}_{\alpha_{1}} \ldots \vec{y}_{\alpha_{s}} R\left(a_{s+1}\right)\right), b, a_{1}, \ldots, a_{s+1} \in R S^{\prime}\left(X_{\Lambda} \cup Y_{\Lambda}\right)$, we have

$$
\begin{aligned}
& R(b) R\left(R\left(a_{1}\right) \vec{y}_{\alpha_{1}} R\left(a_{2}\right) \ldots R\left(a_{s}\right) \vec{y}_{\alpha_{s}} R\left(a_{s+1}\right)\right)
\end{aligned}
$$

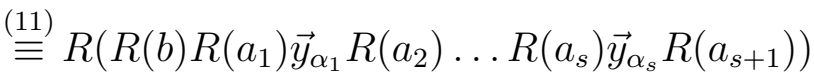

$$
\begin{aligned}
& +R\left(b R\left(R\left(a_{1}\right) \vec{y}_{\alpha_{1}} R\left(a_{2}\right) \ldots R\left(a_{s}\right) \vec{y}_{\alpha_{s}} R\left(a_{s+1}\right)\right)\right) \\
& -R\left(b R\left(a_{1}\right) \vec{y}_{\alpha_{1}} R\left(a_{2}\right) \ldots R\left(a_{s}\right) \vec{y}_{\alpha_{s}} R\left(a_{s+1}\right)\right) \\
& \stackrel{(\underline{8})}{=} R\left(R(b) R\left(a_{1}\right) \vec{y}_{\alpha_{1}} R\left(a_{2}\right) \ldots R\left(a_{s}\right) \vec{y}_{\alpha_{s}} R\left(a_{s+1}\right)\right) \\
& \stackrel{\text { 1117) }}{\equiv} R\left(R\left(R(b) a_{1}+b R\left(a_{1}\right)-b a_{1}\right) \vec{y}_{\alpha_{1}} R\left(a_{2}\right) \ldots R\left(a_{s}\right) \vec{y}_{\alpha_{s}} R\left(a_{s+1}\right)\right) \\
& \stackrel{\underline{\underline{\underline{8}}}}{=} R\left(R(b) a_{1}+b R\left(a_{1}\right)-b a_{1}\right) \vec{y}_{\alpha_{1}} R\left(a_{2}\right) \ldots R\left(a_{s}\right) \vec{y}_{\alpha_{s}} R\left(a_{s+1}\right) \quad \bmod (S, w) \text {. } \\
& R(b) R\left(R\left(a_{1}\right) \vec{y}_{\alpha_{1}} R\left(a_{2}\right) \ldots R\left(a_{s}\right) \vec{y}_{\alpha_{s}} R\left(a_{s+1}\right)\right) \\
& \stackrel{(\underline{\underline{\underline{8}}}}{=} R(b) R\left(a_{1}\right) \vec{y}_{\alpha_{1}} R\left(a_{2}\right) \ldots R\left(a_{s}\right) \vec{y}_{\alpha_{s}} R\left(a_{s+1}\right) \\
& \stackrel{\text { 111) }}{\equiv} R\left(R(b) a_{1}+b R\left(a_{1}\right)-b a_{1}\right) \vec{y}_{\alpha_{1}} R\left(a_{2}\right) \ldots R\left(a_{s}\right) \vec{y}_{\alpha_{s}} R\left(a_{s+1}\right) \quad \bmod (S, w) \text {. }
\end{aligned}
$$

Thus, the corresponding composition of inclusion is trivial modulo $(S, w)$.

Let us calculate a composition of inclusion between (91) and (11). Let $\vec{x}_{\alpha} \in C(X)$, $a, b, c \in R S^{\prime}\left(X_{\Lambda} \cup Y_{\Lambda}\right), w=R(a) R\left(R(b) \vec{x}_{\alpha} R(c)\right)$. Modulo $(S, w)$ we have

$$
\begin{aligned}
& R(a) R\left(R(b) \vec{x}_{\alpha} R(c)\right) \stackrel{\stackrel{(9)}{=}}{=} R(a) R\left(R(b) \vec{x}_{\alpha} c+b \vec{x}_{\alpha} R(c)-b \vec{x}_{\alpha} c\right) \\
& \stackrel{(\underline{111})}{\equiv} R\left(R(a) R(b) \vec{x}_{\alpha} c+R(a) b \vec{x}_{\alpha} R(c)-R(a) b \vec{x}_{\alpha} c\right) \\
& +R\left(a R\left(R(b) \vec{x}_{\alpha} c+b \vec{x}_{\alpha} R(c)-b \vec{x}_{\alpha} c\right)\right)-R\left(a R(b) \vec{x}_{\alpha} c+a b \vec{x}_{\alpha} R(c)-a b \vec{x}_{\alpha} c\right) .
\end{aligned}
$$

For the first summand of the RHS of (12), we obtain

$$
R\left(R(a) R(b) \vec{x}_{\alpha} c\right) \stackrel{\text { (111) }}{=} R\left(R(R(a) b+a R(b)-a b) \vec{x}_{\alpha} c\right) \quad \bmod (S, w) .
$$

Also, modulo $(S, w)$, we have

$$
\begin{aligned}
& R(a) R\left(R(b) \vec{x}_{\alpha} R(c)\right) \stackrel{(11)}{\equiv} R\left(R(a) R(b) \vec{x}_{\alpha} R(c)\right. \\
& \left.+a R\left(R(b) \vec{x}_{\alpha} R(c)\right)-a R(b) \vec{x}_{\alpha} R(c)\right) \stackrel{(\underline{\underline{\underline{9}}}}{=} R\left(R(a) R(b) \vec{x}_{\alpha} R(c)\right) \\
& \quad+R\left(a R\left(R(b) \vec{x}_{\alpha} c+b \vec{x}_{\alpha} R(c)-b \vec{x}_{\alpha} c\right)\right)-R\left(a R(b) \vec{x}_{\alpha} R(c)\right) .
\end{aligned}
$$

Further, the first summand of the RHS of (14) is congruent modulo $(S, w)$ to

$$
\begin{aligned}
& R\left(R(a) R(b) \vec{x}_{\alpha} R(c)\right) \stackrel{(11)}{\equiv} R\left(R(R(a) b+a R(b)-a b) \vec{x}_{\alpha} R(c)\right) \\
& \stackrel{\underline{\equiv} \equiv}{\equiv} R\left(R(a) b \vec{x}_{\alpha} R(c)+a R(b) \vec{x}_{\alpha} R(c)-a b \vec{x}_{\alpha} R(c)\right) \\
&+R\left(R(R(a) b+a R(b)-a b) \vec{x}_{\alpha} c\right)-R\left(R(a) b \vec{x}_{\alpha} c+a R(b) \vec{x}_{\alpha} c-a b \vec{x}_{\alpha} c\right) .
\end{aligned}
$$


Note that substitution of (15) into (14) gives exactly the same as the substitution of (13) into (12). Compositions of inclusion between (10) and (11) are trivial by similar calculations. Theorem is proved.

Corollary 1. The quotient $A$ of $R \operatorname{As}\left\langle X_{\Lambda} \cup Y_{\Lambda}\right\rangle$ by $\operatorname{Id}(S)$ is the universal enveloping associative RB-algebra of the Lie algebra $\hat{L}$ with the RB-operator $R$. Moreover, $\hat{L}$ injectively embeds into $A^{(-)}$.

Proof. The RB-identity (1) when at least one of arguments lies in $S\left(X_{\Lambda}\right) \cup S\left(Y_{\Lambda}\right)$ follows from (15)-(7), (10) and the fact that the space $\mathbb{k} X_{\Lambda}$ is a subalgebra in $\hat{L}$. Within (11), we get an asssociative RB-algebra structure on $A$. By (5)-(7), we have that $A$ is enveloping of $\hat{L}$ for both: the Lie bracket [,] and the action of $R$.

Let us prove that $A$ is the universal enveloping one. At first, $A$ is generated by $\hat{L}$. At second, all elements from $S$ are identities in the universal enveloping associative RBalgebra $U_{R B}(\hat{L})$. Indeed, (5) are enveloping conditions for the product, (11) is the RBidentity. Let us show by induction that (6) and (7) follow from the enveloping conditions for the action of $R$ on $L^{\prime}$ and RB-identity. Suppose that we have proved that $R\left(\vec{y}_{\alpha}\right)=\vec{y}_{\alpha}$ and $R\left(\vec{y}_{\beta}\right)=\vec{y}_{\beta}$, then

$$
\vec{y}_{\alpha} \vec{y}_{\beta}=R\left(\vec{y}_{\alpha}\right) R\left(\vec{y}_{\beta}\right)=R\left(R\left(\vec{y}_{\alpha}\right) \vec{y}_{\beta}+\vec{y}_{\alpha} R\left(\vec{y}_{\beta}\right)-\vec{y}_{\alpha} \vec{y}_{\beta}\right)=R\left(\vec{y}_{\alpha} \vec{y}_{\beta}\right)
$$

Analogously we deduce $R\left(\vec{x}_{\alpha}\right)=0$ for any $\vec{x}_{\alpha} \in S(X)$.

The relations (10) follow from (7) and (11) immediately. Now we state (9):

$$
\begin{aligned}
& 0=R(a) R\left(\vec{x}_{\alpha}\right) R(b)=R\left(R(a) \vec{x}_{\alpha}-a \vec{x}_{\alpha}\right) R(b) \\
&=R\left(R(a) \vec{x}_{\alpha} R(b)-a \vec{x}_{\alpha} R(b)\right.\left.+R\left(R(a) \vec{x}_{\alpha}-a \vec{x}_{\alpha}\right) b-R(a) \vec{x}_{\alpha} b+a \vec{x}_{\alpha} b\right) \\
&=R\left(R(a) \vec{x}_{\alpha} R(b)-a \vec{x}_{\alpha} R(b)-R(a) \vec{x}_{\alpha} b+a \vec{x}_{\alpha} b\right) .
\end{aligned}
$$

Finally, let us prove (8) by induction on $s$ analogously to (16):

$$
\begin{array}{r}
R\left(a_{1}\right) \vec{y}_{\alpha_{1}} R\left(a_{2}\right) \ldots R\left(a_{s}\right) \vec{y}_{\alpha_{s}} R\left(a_{s+1}\right)=R\left(R\left(a_{1}\right) \vec{y}_{\alpha_{1}} \ldots R\left(a_{s}\right)\right) R\left(\vec{y}_{\alpha_{s}} R\left(a_{s+1}\right)\right) \\
=R\left(R\left(a_{1}\right) \vec{y}_{\alpha_{1}} R\left(a_{2}\right) \ldots R\left(a_{s}\right) \vec{y}_{\alpha_{s}} R\left(a_{s+1}\right)\right) .
\end{array}
$$

Hence, the quotient of $R \operatorname{As}\left\langle X_{\Lambda} \cup Y_{\Lambda}\right\rangle$ by $\operatorname{Id}(S)$ is the universal enveloping associative RB-algebra for $\hat{L}$. By Theorems 3 and 4 we get the injectivity of embedding $\hat{L}$ into $A^{(-)}$. Corollary is proved.

Remark. Analogously to Corollary 1, one can find the linear basis of the universal enveloping associative RB-algebra for a Lie algebra endowed with an RB-operator $R$ of nonzero weight $\lambda$ satisfying $R^{2}=-\lambda R$.

Corollary 2. Any post-Lie algebra injectively embeds into its universal enveloping postassociative algebra.

ProOf. Let $L$ be a post-Lie algebra. By Theorem 2, $L$ can be injectively embedded into $\hat{L}^{(R)}$ with the RB-operator $R$ of weight -1 . Then, by Corollary 1 , we embed the Lie RB-algebra $\hat{L}$ into the associative algebra $A$ with the RB-operator $P$. Thus, the subalgebra (in postalgebra sense) $T$ in $A^{(P)}$ generated by the set $L^{\prime}=\operatorname{Span}\left\{x_{\alpha}-y_{\alpha} \mid\right.$ $\alpha \in \Lambda\}$ is an (injective) enveloping postassociative algebra of initial post-Lie algebra $L$.

The question whether the pair (postLie, postAs) is a PBW-pair is still open. 


\section{Acknowledgements}

The author expresses his gratitude to P. Kolesnikov for the useful comments and remarks.

This work was supported by the Austrian Science Foundation FWF grant P28079.

\section{References}

[1] M. Aguiar, Pre-Poisson algebras, Lett. Math. Phys. 54 (2000) 263-277.

[2] C. Bai, O. Bellier, L. Guo, X. Ni, Splitting of operations, Manin products, and Rota-Baxter operators, Int. Math. Res. Notices 3 (2013) 485-524.

[3] C. Bai, L. Guo, X. Ni, $\mathcal{O}$-operators on associative algebras, associative Yang-Baxter equations and dendriform algebras, Conference Proceedings Quantized Algebra and Physics (2012) 10-51.

[4] G. Baxter, An analytic problem whose solution follows from a simple algebraic identity, Pacific J. Math. 10 (1960) 731-742.

[5] A.A. Belavin, V.G. Drinfel'd, Solutions of the classical Yang-Baxter equation for simple Lie algebras, Funct. Anal. Appl. 16 (3) (1982) 159-180.

[6] L.A. Bokut, Yu. Chen, X. Deng, Gröbner-Shirshov bases for Rota-Baxter algebras, Sib. Math. J. 51 (6) (2010) 978-988.

[7] P. Cartier, On the structure of free Baxter algebras, Adv. Math. 9 (1972) 253-265.

[8] Yu. Chen, Q. Mo, Embedding dendriform algebra into its universal enveloping Rota-Baxter algebra, Proc. Amer. Math. Soc. 139 (12) (2011) 4207-4216.

[9] I. Dokas, Pre-Lie algebras in positive characteristic, J. Lie Theory 23 (4) (2013) 937-952.

[10] V. Dotsenko, P. Tamaroff, Endofunctors and Poincaré-Birkhoff-Witt theorems, arXiv:1804.06485 [math.CT].

[11] K. Ebrahimi-Fard, Loday-type algebras and the Rota-Baxter relation, Lett. Math. Phys. 61 (2002) 139-147.

[12] K. Ebrahimi-Fard, L. Guo, Rota-Baxter algebras and dendriform algebras, J. Pure Appl. Algebra 212 (2) (2008) 320-339.

[13] M. Gerstenhaber, The cohomology structure of an associative ring, Ann. of Math. 78 (1963) 267-288.

[14] V. Ginzburg, M. Kapranov, Koszul duality for operads, Duke Math. J. 76 (1) (1994) 203-272. 
[15] I.Z. Golubchik, V.V. Sokolov, Generalized operator Yang-Baxter equations, integrable ODEs and nonassociative algebras, J. Nonlinear Math. Phys. 7 (2) (2000) 184-197.

[16] V.Yu. Gubarev, Free Lie Rota-Baxter algebras, Sib. Math. J. 57 (5) (2016) 809-818.

[17] V. Gubarev, Universal enveloping commutative Rota-Baxter algebras of pre- and post-commutative algebras, Axioms 6 (4) (2017) 1-33.

[18] V. Gubarev, Universal enveloping associative Rota-Baxter algebras of preassociative and postassociative algebras, arXiv:1708.00168.

[19] V. Gubarev, Universal enveloping Lie Rota-Baxter algebras of pre-Lie and post-Lie algebras, arXiv:1708.06747.

[20] V. Gubarev, P. Kolesnikov, Embedding of dendriform algebras into Rota-Baxter algebras, Cent. Eur. J. Math. 11 (2) (2013) 226-245.

[21] V. Gubarev, P. Kolesnikov, Operads of decorated trees and their duals, Comment. Math. Univ. Carolin. 55 (4) (2014) 421-445.

[22] V. Gubarev, P. Kolesnikov, Groebner-Shirshov basis of the universal enveloping Rota-Baxter algebra of a Lie algebra, J. Lie Theory 27 (3) (2017) 887-905.

[23] L. Guo, An Introduction to Rota-Baxter Algebra, Intern. Press, Somerville, MA; Higher education press, Beijing, 2012.

[24] L. Guo, W. Keigher, Baxter algebras and shuffle products, Adv. Math. 150 (2000) $117-149$.

[25] L. Guo, W. Sit, and R. Zhang, Differential type operators and Gröbner-Shirshov bases, J. Symb. Comput. 52 (2013) 97-123.

[26] P. Kolesnikov, Gröbner-Shirshov bases for pre-associative algebras, Commun. Algebra 45 (12) (2017) 5283-5296.

[27] J.-L. Loday, Cup-product for Leibniz cohomology and dual Leibniz algebras, Math. Scand. 77 (2) (1995) 189-196.

[28] J.-L. Loday, Dialgebras, Dialgebras and related operads, 1-61, Springer-Verl., Berlin, 2001.

[29] J.-L. Loday, On the algebra of quasi-shuffles, Manuscripta Math. 123 (2007) 79-93.

[30] J.-L. Loday, T. Pirashvili, Universal enveloping algebras of Leibniz algebras and (co)homology, Math. Ann. 296 (1993) 139-158.

[31] J.-L. Loday, M. Ronco, Trialgebras and families of polytopes, Comtep. Math. 346 (2004) 369-398. 
[32] A.A. Mikhalev, I.P. Shestakov, PBW-pairs of varieties of linear algebras, Commun. Algebra 42 (2) (2014) 667-687.

[33] J. Qiu, Yu. Chen, Gröbner-Shirshov bases for Lie $\Omega$-algebras and free Rota-Baxter Lie algebras, J. Algebra Appl. 16 (2) (2017) 175-190.

[34] G.-C. Rota, Baxter algebras and combinatorial identities I, Bull. Amer. Math. Soc. 75 (1969) 325-329.

[35] M.A. Semenov-Tyan-Shanskii, What is a classical $r$-matrix? Funct. Anal. Appl. 17 (4) (1983) 259-272.

[36] B. Vallette, Homology of generalized partition posets, J. Pure Appl. Algebra 208 (2) (2007) 699-725.

[37] E.B. Vinberg, Homogeneous cones, Sov. Math. Dokl. 1 (1960) 787-790.

Vsevolod Gubarev

University of Vienna

Oskar-Morgenstern-Platz 1, 1090 Vienna, Austria

Sobolev Institute of mathematics

Acad. Koptyug ave. 4, 630090 Novosibirsk, Russia

e-mail: vsevolod.gubarev@univie.ac.at 